\title{
Temporal Purity and Quantum Interference of Single Photons from Two Independent Cold Atomic Ensembles
}

\author{
Peng Qian, ${ }^{1}$ Zhenjie Gu, ${ }^{1}$ Rong Cao, ${ }^{1}$ Rong Wen, ${ }^{1}$ Z. Y. Ou, ${ }^{1,3}$ J. F. Chen, ${ }^{1,4,}$ and Weiping Zhang ${ }^{2,4, \dagger}$ \\ ${ }^{1}$ Quantum Institute of Light and Atoms, Department of Physics, East China Normal University, \\ Shanghai 200241, People's Republic of China \\ ${ }^{2}$ Department of Physics and Astronomy, Shanghai Jiao Tong University, Shanghai 200240, People's Republic of China \\ ${ }^{3}$ Department of Physics, Indiana University-Purdue University Indianapolis, 402 North Blackford Street, \\ Indianapolis, Indiana 46202, USA \\ ${ }^{4}$ Collaborative Innovation Center of Extreme Optics, Shanxi University, Taiyuan, Shanxi 030006, People's Republic of China
}

(Received 23 February 2016; revised manuscript received 29 April 2016; published 1 July 2016)

\begin{abstract}
The temporal purity of single photons is crucial to the indistinguishability of independent photon sources for the fundamental study of the quantum nature of light and the development of photonic technologies. Currently, the technique for single photons heralded from time-frequency entangled biphotons created in nonlinear crystals does not guarantee the temporal-quantum purity, except using spectral filtering. Nevertheless, an entirely different situation is anticipated for narrow-band biphotons with a coherence time far longer than the time resolution of a single-photon detector. Here we demonstrate temporally pure single photons with a coherence time of $100 \mathrm{~ns}$, directly heralded from the time-frequency entangled biphotons generated by spontaneous four-wave mixing in cold atomic ensembles, without any supplemented filters or cavities. A near-perfect purity and indistinguishability are both verified through Hong-Ou-Mandel quantum interference using single photons from two independent cold atomic ensembles. The time-frequency entanglement provides a route to manipulate the pure temporal state of the single-photon source.
\end{abstract}

DOI: 10.1103/PhysRevLett.117.013602

The purity of the quantum state of a single photon is a prerequisite of its indistinguishability with single photons from other independent sources, and the latter is an essential basis for the realization of a scalable quantum network with distant and independent nodes [1-5]. Furthermore, the temporal purity of a single photon is crucial to the development of photonic technologies for quantum information science [6,7]. The traditional method to produce indistinguishable single photons is heralding time-frequency entangled biphotons generated from spontaneous parametric down-conversion (SPDC) in a nonlinear crystal which is pumped by ultrashort pulses $[4,5,8]$. In recent decades many new physical systems have been developed [9-13] to obtain pure single photons without time-frequency entanglement built in.

In the community of quantum communication, SPDC in $\chi^{(2)}$ nonlinear media is still the preferable way to produce entangled biphotons because of its simplicity in the operation and the potential for on-chip integration and scaling up [14-16]. However, the intrinsic feasible phase matching condition of SPDC crystal allows an extremely broad range of temporal modes. Therefore, the typical temporal coherence time of the photon source is of femtosecond scale. Compared to the time response of most commercial single-photon detectors, which is about $1 \mathrm{~ns}$, this temporal coherence time is so short that the trigger photon of the heralded single photon is measured with a large time uncertainty. This time uncertainty damages the temporal quantum purity of the single-photon source. To circumvent the time uncertainty problem due to the slowness of the detectors, a common practice is to use external spectral filtering including passive filtering with narrowband filters [4,5,17] and active filtering with an optical cavity [18]. In this case, the temporal state of the single photons relies on the spectral property of the external passive filter or optical cavity, which is monolithic and unavailable to be manipulated. On the other hand, to avoid filtering and produce a bright single-photon source, the spectral correlation can be removed through engineering the SPDC source [19-23]. The factorable joint amplitude for the signal and idler photons is necessary to guarantee a pure single-photon state. Therefore, for SPDC source, the temporal-quantum state purity of the heralded single photons, and the time-frequency entanglement of the corresponding photon pairs cannot be simultaneously satisfied. However, the time-frequency entanglement of biphotons is a great advantage in manipulating the singlephoton state via nonlocal spectral modulation [24-26]. If one wants the temporal purity and the time-frequency entanglement both maintained in the photon source, one needs a long temporal coherence so that the biphoton coherence time is much longer than the trigger response time [27]. Subsequently, how to experimentally attain a perfect temporal purity of single photons directly from the 
time-frequency entangled sources of biphotons without any external filtering remains an open question.

Generating narrow-band paired photons from $\chi^{(3)}$ in atomic ensembles is a technique that has developed quickly in recent years, and the paired photons are entangled in the time-frequency domain [28]. With a cold atomic ensemble as the nonlinear medium, the dephasing rate of $\chi^{(3)}$ is comparable to the atomic natural linewidth [29-34]. In particular, the biphotons generated from spontaneous fourwave mixing (SFWM) in cold atomic ensembles have a coherence time exceeding $1 \mu \mathrm{s}[35,36]$ with the electromagnetically induced transparency (EIT) effect [37]. The EIT slow light effect reduces the group velocity of the signal photons and, hence, extends the coherence time to $100 \mathrm{~ns}$ or more. Different from the external spectral filtering in the SPDC scheme, the EIT effect governs not only the transmission of the photons but mainly the phase matching condition of the FWM process. The phase matching condition, therefore, is dependent on the atomic ensemble and the coupling field and can be manipulated temporally much more easily [38-42]. In this Letter, we show that the heralded single photon with a coherence time of $100 \mathrm{~ns}$ directly heralded from time-frequency entangled biphotons generated from cold atom clouds is in a temporally pure quantum state. The purity and indistinguishability are both verified through the observation of a HOM interference [43] between independent single photons from separate cold atom clouds with visibility up to $80 \%$ including the multiphoton events. It is the first experimental work to demonstrate that a single photon heralded from time-frequency entangled biphotons is in a pure temporal-quantum state. The time-frequency entanglement of the paired photons provides a route to reshape the single photons but without damaging its temporal purity via nonlocal spectral modulation.

A temporally pure single-photon state is a coherent superposition of a single photon in a different frequency:

$$
|1(T)\rangle=\int d \Omega \varphi(\Omega) e^{i \Omega T} \hat{a}^{\dagger}(\bar{\omega}+\Omega)|0\rangle,
$$

where $\bar{\omega}$ is the center frequency, and the spectral function $\varphi(\Omega)$ satisfies the normalization relation $\int|\varphi(\Omega)|^{2} d \Omega=1$. Equation (1) describes a single-photon wave packet of temporal profile $h(t)=(1 / 2 \pi) \int d \Omega \varphi(\Omega) e^{-j \Omega(t-T)}$, which is centered around $t=T$. Typically, the single photons are produced by nonclassical spectral correlated photon pairs (signal and idler photons) on which an annihilation operator of the idler field is applied to obtain the signal field as the heralded single photons. If the arrival time of the idler cannot be resolved exactly by detectors, an equivalent uncertainty $\Delta T$ should be considered and, therefore, cast the single photon into a nonpure state which should be described by purity [27]

$$
\begin{aligned}
\operatorname{Tr}\left\{\hat{\rho}^{2}(\Delta T)\right\} & =\frac{1}{(\Delta T)^{2}} \int_{\Delta T} d T d T^{\prime}\left|\left\langle 1(T) \mid 1\left(T^{\prime}\right)\right\rangle\right|^{2} \\
& =\int d \Omega d \Omega^{\prime}\left|\varphi(\Omega) \varphi\left(\Omega^{\prime}\right)\right|^{2} \operatorname{sinc}^{2} \frac{\left(\Omega-\Omega^{\prime}\right) \Delta T}{2}
\end{aligned}
$$

in which the purity of the quantum state $\gamma \equiv \operatorname{Tr}\left\{\hat{\rho}^{2}(\Delta T)\right\}$. For the SPDC source without spectral filtering, the sinc function in Eq. (2) is always 0 . This is because $\Delta T$ determined by the time resolution of the detectors is much longer than the coherence time of the SPDC photons. Nevertheless, an entirely different situation is anticipated for narrow-band biphotons with a coherence time far longer than the time resolution of the single-photon detector. Hundreds of nanoseconds of temporal coherence give a narrow linewidth of $<10 \mathrm{MHz}$, and the time uncertainty caused by detection $(\sim 1 \mathrm{~ns})$ is small enough to make the sinc function in Eq. (2) close to unity. Therefore, the purity $\gamma$ approaches unity, and the time-frequency entanglement do not damage the purity through the heralding. The physics can be explained as follows: If the coherence time of the spectral correlated paired photons is long enough so that different temporal modes of the photon pairs can be resolved by the detectors, the temporal state of the signal photon is a superposition of different temporal eigenstates. In this way, the time-frequency entanglement does no harm to the temporal purity of the single photon but rather provides a way to shape its temporal states.

To verify the single-photon purity, we experimentally demonstrate the HOM interference from two independent single photons produced from the SFWM process in separate cold atom clouds. The cold atom clouds for the generation of heralded single photons are prepared by twodimensional (2D) ${ }^{85} \mathrm{Rb}$ magneto-optical traps (MOT1 and MOT2 in Fig. 1), with a temperature of about $100 \mu \mathrm{K}$ $[36,44]$. In both MOTs, with the presence of a counterpropagating pump (pump, $780 \mathrm{~nm}$ ) and coupling (coupling, $795 \mathrm{~nm}$ ) beams which are aligned at $3^{\circ}$ from the longitudinal axis, the Stokes $\left(\omega_{s}\right.$, as the idler) and anti-Stokes $\left(\omega_{a s}\right.$, as the signal) photon pairs [32] are emitted from the atom clouds in opposite directions. The biphotons are generated from SFWM in the double- $\Lambda$ four-level atomic system, with the same energy level diagram as Ref. [36]. The atoms are prepared in the ground state $|1\rangle=5 S_{1 / 2}$, $F=2$, pumped by a continuous pump laser which is blue detuned from the transition $|1\rangle \leftrightarrow|4\rangle=5 P_{3 / 2}, F=3$ by $\Delta_{p}=146 \mathrm{MHz}$. Since the coupling laser is on resonance with the transition $|2\rangle=5 S_{1 / 2}, \quad F=3 \leftrightarrow|3\rangle=5 P_{1 / 2}$, $F=3$, the anti-Stokes photons undergo a resonant EIT effect. We run the experiment periodically with a MOT time of $4.5 \mathrm{~ms}$, followed by a biphoton generation window of $0.5 \mathrm{~ms}$ at each period cycle. Different from the pulsed pumping scheme, the pump and coupling beams are continuous waves, and, thus, the synchronization excitation 


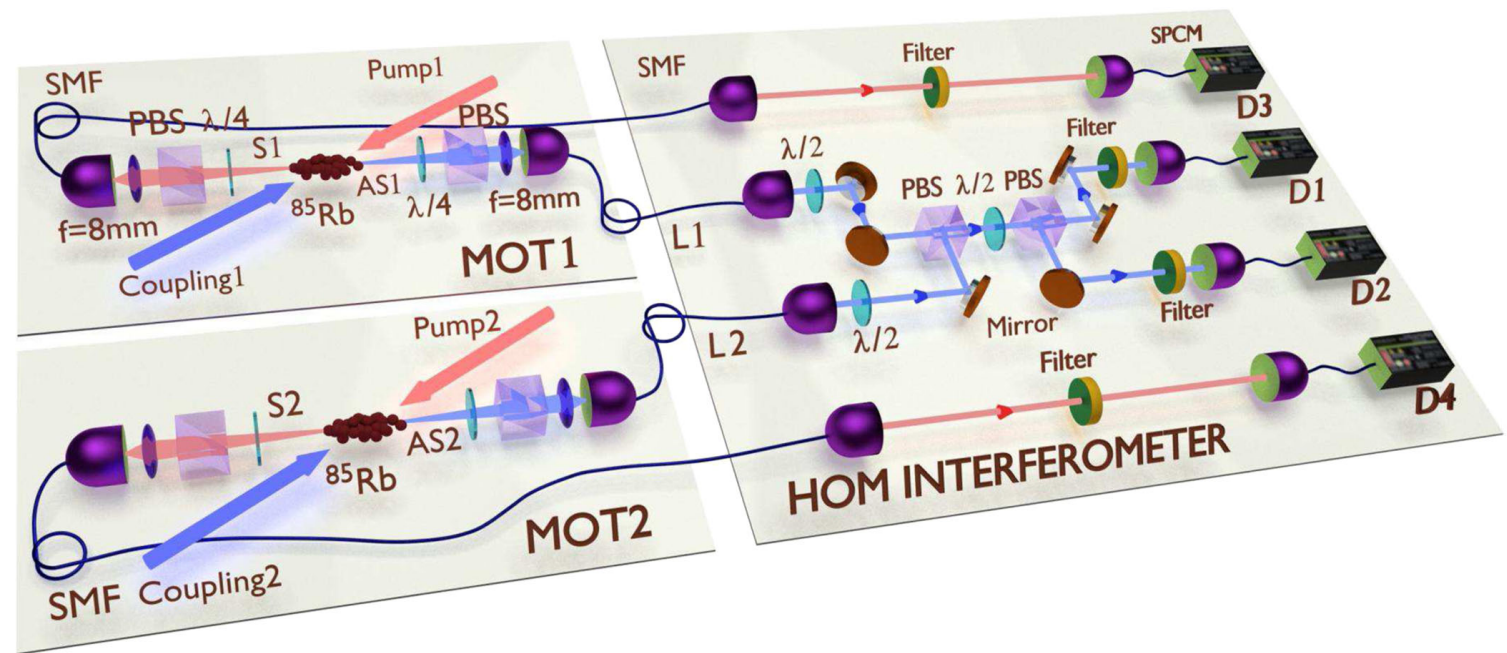

FIG. 1. Experimental setup. MOT1 and MOT2 are two separate and independently running magneto-optical traps. For each MOT, we apply counterpropagating pump and coupling beams, and through backscattering, we collect Stokes (S) and anti-Stokes (AS) with single-mode fibers. Through polarization-maintained SMF $L 1$ and $L 2$, the photons are directed to the Hong-Ou-Mandel (HOM) interferometer table.

of two atom clouds is not necessary. For each MOT, the Stokes and anti-Stokes photons generated from the atom clouds are collected through a pair of polarizers followed by polarization-maintained SMFs, and the anti-Stokes photons are directed to a beam splitter configuration as shown in Fig. 1. The half-wave plates (HWPs) at two input ends are designed to rotate the independent single-photon beams to be horizontally and vertically polarized, respectively. When the axis of the HWPs located between two polarization beam splitters (PBSs) is oriented $45^{\circ}$ with respect to the horizontal and vertical axes, the second PBS serves as a 50:50 beam splitter where the two independent single-photon beams mix. Otherwise, if the axis of the HWP is aligned with the horizontal or vertical axes, the two beams simply separate at the second PBS and no interference occurs.

With the detection of a Stokes photon, the anti-Stokes field becomes a single-photon state with a temporal wave function amplitude of $h_{1(2)}(\tau)=\int d \Omega \varphi_{1(2)}(\Omega) e^{i \Omega \tau}$ for MOT1 (or MOT2), with $\tau$ as the relative delay of the anti-Stokes photons. The arrival-time difference between two independent Stokes photons is denoted by $\Delta t$. With perfectly matched polarization modes and spatial modes, the fourfold coincidence count rate is given by [45]

$P^{(4)}=\left(T^{2}+R^{2}\right) H_{1} H_{2}[1-\mathcal{V} \Gamma(\Delta t)]+4 T^{2} R^{2} \tilde{g}^{(2)} \frac{H_{1}}{2} \frac{H_{2}}{2}$,

with $H_{1(2)} \equiv \int d \tau\left|h_{1(2)}(\tau)\right|^{2}, \quad \mathcal{V}=2 T R /\left(T^{2}+R^{2}\right)$, and $\Gamma(\Delta t) \equiv \int d \tau\left|h_{1}^{*}(\tau) h_{2}(\tau+\Delta t)\right|^{2} / H_{1} H_{2}$. Obviously, for the 50:50 BS, $\mathcal{V}=100 \%$. In addition, we keep the noise term to include the excess counts created by a nonideal single-photon state in which $\tilde{g}^{(2)}$ is the effective normalized second-order autocorrelation function. The nonzero $\tilde{g}^{(2)}$ is caused by the multiphoton events, and it gives rise to the imperfect HOM dip.
To maximize the visibility of the HOM dip, we carefully match the temporal mode of the independent single photons emitted from two separate MOTs. The single-photon temporal wave function is affected by the effective intensity of the pump and coupling beams, the spatial profile of the pump beam, and the optical depth of the atom cloud. The pump and coupling beams are collimated with a diameter of $1.6 \mathrm{~mm}$. The pump and coupling beam power is around $80 \mu \mathrm{W}$ and $2 \mathrm{~mW}$, respectively; the optical depth is about 20 for both MOTs. All of these parameters are fine-tuned to match the single-photon wave functions produced from the two MOTs. Figure 2(a) shows $h_{1}(\tau)$ and $h_{2}(\tau)$ from MOT1 and MOT2, respectively. The similarity defined as $\int\left[\sqrt{h_{1}(\tau)} \times \sqrt{h_{2}(\tau)}\right] d \tau /\left[\int \sqrt{h_{1}(\tau)} d \tau\right]\left[\int \sqrt{h_{2}(\tau)} d \tau\right]$ is obtained as $97 \% \pm 2 \%$. For both MOTs, the coherence time of the biphoton source, which is defined as the relative time where the coincidence counts decay as $1 / e^{2}$ of the peak value in Fig. 2, is about 100 ns. When measuring the second-order autocorrelation function $g^{(2)}$, we use a measurement window from $\tau=0$ to $\tau=150 \mathrm{~ns}$ with an effective measurement time of $3 \mathrm{~min}$. Our measurements give $g_{1}^{(2)}=0.193 \pm 0.004$ and $g_{2}^{(2)}=0.196 \pm 0.004$ for MOT1 and MOT2. In Fig. 2(b), the black squares display the fourfold coincidence count rate as a function of $\Delta t$, with $\tau_{1}$ and $\tau_{2}$ integrated over a temporal window of $150 \mathrm{~ns}$. With the time step of $\Delta t$ set as $10 \mathrm{~ns}$, the result shows an $80 \%$ visibility of a HOM dip at $\Delta t=0$. Equation (3) gives us the theoretical results as plotted by the blue solid curve in Fig. 2(b), with $\tau_{1}, \tau_{2}$ integrated. For simplicity, we consider $g_{1}^{(2)} \approx g_{2}^{(2)}$, and, therefore, $\tilde{g}^{(2)}=0.2$ in Eq. (3). Therefore, after taking into account the noise term aroused from the nonzero $\tilde{g}^{(2)}$, the theory predicts about $90 \%$ visibility. The discrepancy between the theory and the experimental results is caused by the mismatch of the 

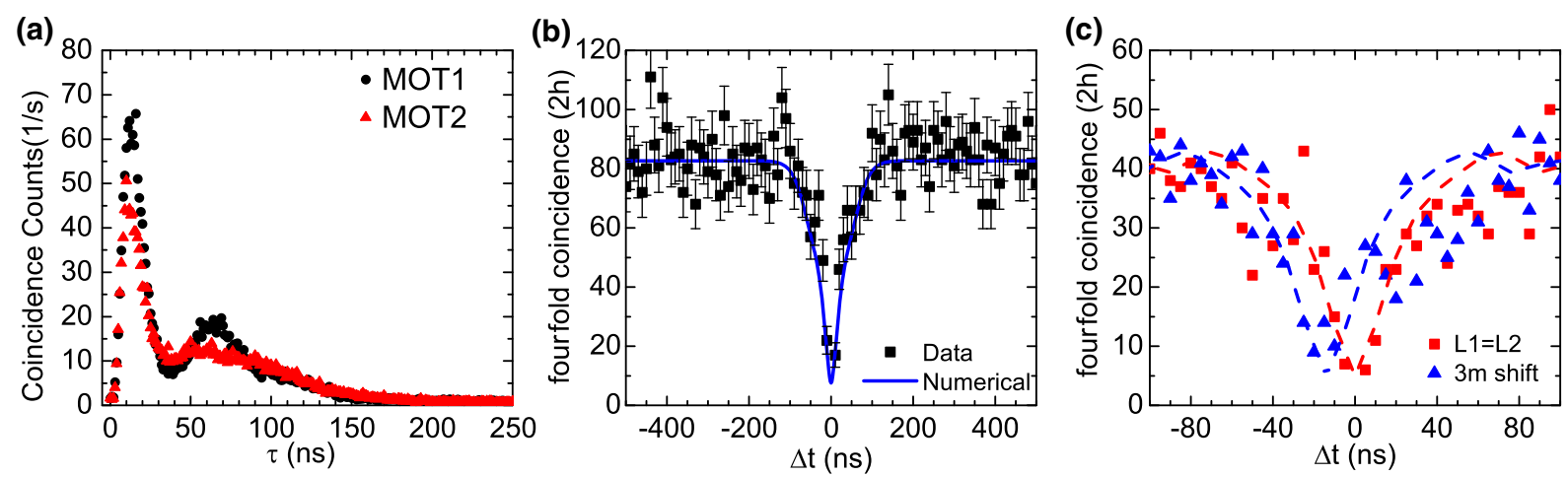

FIG. 2. Observation of HOM interference of single photons from independent MOTs with OD = 20. (a) Coincidence measurement of S1,AS1 and S2,AS2 indicating the probability amplitudes of the single-photon wave function of MOT1 (black circles) and MOT2 (red triangles). (b) Fourfold photon coincidence count rate in $2 \mathrm{~h}$ of effective measurement time, with $10 \mathrm{~ns}$ as a time step. The dark squares with error bars plot the experimental data for the symmetric photon paths $(L 1=L 2)$, and the blue solid line shows the theoretical curves according to Eq. (3), with $h_{1}(t)$ and $h_{2}(t)$ fitting the coincidence results of (a). (c) Fourfold photon coincidence count rate in $2 \mathrm{~h}$ of the effective measurement time with $5 \mathrm{~ns}$ as a time step. The red squares and blue triangles mark the experimental data with $L 1=L 2$ and $L 1=L 2+3 \mathrm{~m}$ (with 3-m-fiber inserted). They are guided by the red and blue dashed lines, respectively.

polarization, spatial, and temporal modes of the independent sources.

The arrival time difference between the two independent pairs of photons $\Delta t$ is equivalent to the path delay between the two arms of the HOM interferometer. To confirm this, we insert a 3-m polarization maintaining fiber into the output path of the anti-Stokes beam of MOT2 $[L 2 \neq L 1$ in Fig. 1(c)]. As expected, the asymmetric path arrangement simply causes an approximate $10 \mathrm{~ns}$ shift of the HOM dip at the fourfold coincidence measurement without a severe change of visibility, as shown by the blue triangles in Fig. 2(c).

According to Eq. (2), the purity of the heralded singlephoton state is determined by the time uncertainty $\Delta T$, the minimum time step of $\Delta t$. For high purity, the time uncertainty $\Delta T$ should be compared with the coherence time, which is inversely proportional to the single-photon bandwidth (BW), and the product $\mathrm{BW} \Delta T$ determines the purity. The visibility of the HOM interference gives the lower bound of the single-photon purity [20]. From $h_{1}(\tau)$, $h_{2}(\tau)$, we can obtain the bandwidth of the heralded single photons $[\mathrm{BW}=10 \mathrm{MHz}$ for Fig. 2(a)]. Therefore, in Fig. 3, we plot the average purity as a function of $\mathrm{BW} \Delta T$. In this figure, we correct the visibility after taking into account the multipair contribution due to $\tilde{g}^{(2)} \neq 0$. The experimental result is denoted as black squares. For comparison, we show the theoretical curves simulated from Eq. (2), with $\varphi(\Omega)$ calculated from the actual SFWM process with the actual parameters [47]. As expected, the experimental values for the purity locate around the theoretical curves describing a mixed singlephoton state, which indicates a reduction with a growing value of BW $\Delta T$. From the theory and our experimental results, when $\mathrm{BW} \Delta T \leq 0.1$, the single-photon state can be considered as pure. The heralded single photons generated from cold atomic ensembles is in a pure temporal-quantum state without filters or damaging the time-frequency entanglement.

In conclusion, we observe the HOM interference from two independent single-photon sources with visibility as high as $80 \%$. When the time response of the detectors is fast enough to resolve the biphoton coherence time, the temporal purity of the heralded single photons produced from the time-frequency entangled biphotons approaches unity. With the entanglement, purity, and indistinguishability all kept, the narrow-band single-photon source generated from cold atomic ensembles is useful in quantum information processing and quantum networks. For example, through maintaining the entanglement in the timefrequency domain, the heralded single-photon source from cold atomic ensembles can be manipulated through nonlocal spectral modulation. Also, with single-photon purity and indistinguishability, entanglement can be encoded into

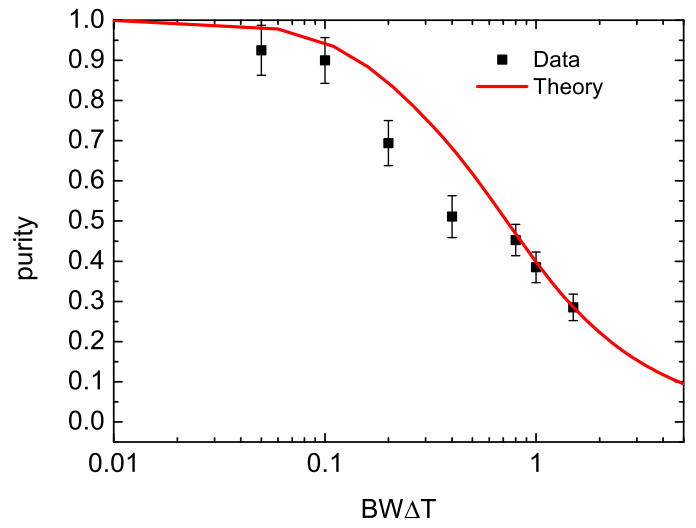

FIG. 3. Purity of a single photon as a function of BW $\Delta T$. BW is the bandwidth of the emitted paired photon, and $\Delta T$ is the detector response time. The black squares with error bar denote the experimental data, and the red solid line denotes the numerical result of Eq. (2). 
noninteracting particles which share no common past through entanglement swapping between completely autonomous media.

We acknowledge P. Chen for his help in the dataprocessing program used in the fourfold coincidence measurement. This work is supported by National Key Research Program of China under Grant No. 2016YFA0302000 and Grant No. 2011CB921604, the National Natural Science Foundation of China (NSFC) through the major research plan (Precision Measurement Physics Grant No. 91436211), NSFC Key Research Program No. 11234003, Young Scientists Research Program No. 11204086, and the Shanghai Natural Science Foundation Grant No. 16ZR1448200.

P. Q. and Z. G. contributed equally to this work.

jfchen@phy.ecnu.edu.cn †wpzhang@phy.ecnu.edu.cn

[1] M. Zukowski, A. Zeilinger, M. A. Horne, and A. K. Ekert, Phys. Rev. Lett. 71, 4287 (1993).

[2] J.-W. Pan, D. Bouwmeester, H. Weinfurter, and A. Zeilinger, Phys. Rev. Lett. 80, 3891 (1998).

[3] E. Knill, R. Laflamme, and G. J. Milburn, Nature (London) 409, 46 (2001).

[4] R. Kaltenbaek, B. Blauensteiner, M.Żukowski, M. Aspelmeyer, and A. Zeilinger, Phys. Rev. Lett. 96, 240502 (2006).

[5] T. Yang, Q. Zhang, T.-Y. Chen, S. Lu, J. Yin, J.-W. Pan, Z.-Y. Wei, J.-R. Tian, and J. Zhang, Phys. Rev. Lett. 96, 110501 (2006).

[6] B. Brecht, D. V. Reddy, C. Silberhorn, and M. G. Raymer, Phys. Rev. X 5, 041017 (2015).

[7] A. I. Lovovsky and M. G. Raymer, Rev. Mod. Phys. 81, 299 (2009).

[8] J.-W. Pan, Z.-B. Chen, C.-Y. Lu, H. Weinfurter, A. Zeilinger, and M. Żukowski, Rev. Mod. Phys. 84, 777 (2012).

[9] R. B. Patel, A. J. Bennett, I. Farrer, C. A. Nicoll, D. A. Ritchie, and A. J. Shields, Nat. Photonics 4, 632 (2010).

[10] X. Ding et al., Phys. Rev. Lett. 116, 020401 (2016).

[11] J. Beugnon, M. P. A. Jones, J. Dingjan, B. Darquié, G. Messin, A. Browaeys, and P. Grangier, Nature (London) 440, 779 (2006).

[12] T. Chanelière, D. N. Matsukevich, S. D. Jenkins, S.-Y. Lan, R. Zhao, T. A. B. Kennedy, and A. Kuzmich, Phys. Rev. Lett. 98, 113602 (2007).

[13] H. Bernien, L. Childress, L. Robledo, M. Markham, D. Twitchen, and R. Hanson, Phys. Rev. Lett. 108, 043604 (2012).

[14] S. V. Zhukovsky, L. G. Helt, D. Kang, P. Abolghasem, A. S. Helmy, and J. E. Sipe, Phys. Rev. A 85, 013838 (2012).

[15] S. M. Spillane, M. Fiorentino, and R. G. Beausoleil, Opt. Express 15, 8770 (2007).

[16] L. Sansoni, F. Sciarrino, G. Vallone, P. Mataloni, A. Crespi, R. Ramponi, and R. Osellame, Phys. Rev. Lett. 105, 200503 (2010).

[17] M. Halder, A. Beveratos, N. Gisin, V. Scarani, C. Simon, and H. Zbinden, Nat. Phys. 3, 692 (2007).
[18] J. Yang, X. H. Bao, H. Zhang, S. Chen, C. Z. Peng, Z. B. Chen, and J. W. Pan, Phys. Rev. A 80, 042321 (2009).

[19] W. P. Grice, A. B. U'Ren, and I. A. Walmsley, Phys. Rev. A 64, 063815 (2001).

[20] P. J. Mosley, J. S. Lundeen, B. J. Smith, P. Wasylczyk, A. B. U'Ren, C. Silberhorn, and I. A. Walmsley, Phys. Rev. Lett. 100, 133601 (2008).

[21] P. G. Evans, R. S. Bennink, W. P. Grice, T. S. Humble, and J. Schaake, Phys. Rev. Lett. 105, 253601 (2010).

[22] N. Bruno, A. Martin, T. Guerreiro, B. Sanguinetti, and R. T. Thew, Opt. Express 22, 17246 (2014).

[23] B. Brecht, A. Eckstein, R. Ricken, V. Quiring, H. Suche, L. Sansoni, and C. Silberhorn, Phys. Rev. A 90, 030302(R) (2014).

[24] S. E. Harris, Phys. Rev. A. 78, 021807(R) (2008).

[25] C. Belthangady, C.-S. Chuu, I. A. Yu, G. Y. Yin, J. M. Kahn, and S. E. Harris, Phys. Rev. Lett. 104, 223601 (2010).

[26] S. Sensarn, G. Y. Yin, and S. E. Harris, Phys. Rev. Lett. 103, 163601 (2009).

[27] S. Du, Phys. Rev. A 92, 043836 (2015).

[28] P. Chen, C. Shu, X. Guo, M. M. T. Loy, and S. Du, Phys. Rev. Lett. 114, 010401 (2015).

[29] A. Kuzmich, W. P. Bowen, A. D. Boozer, A. Boca, C. W. Chou, L.-M. Duan, and H. J. Kimble, Nature (London) 423, 731 (2003).

[30] V. Balić, D. A. Braje, P. Kolchin, G. Y. Yin, and S. E. Harris, Phys. Rev. Lett. 94, 183601 (2005).

[31] P. Kolchin, S. Du, C. Belthangady, G. Y. Yin, and S. E. Harris, Phys. Rev. Lett. 97, 113602 (2006).

[32] S. Du, P. Kolchin, C. Belthangady, G. Y. Yin, and S. E. Harris, Phys. Rev. Lett. 100, 183603 (2008).

[33] D.-S. Ding, Z.-Y. Zhou, B.-S. Shi, and G.-C. Guo, Nat. Commun. 4, 2527 (2013).

[34] D.-S. Ding, W. Zhang, Z.-Y. Zhou, S. Shi, B.-S. Shi, and G.-C. Guo, Nat. Photonics 9, 332 (2015).

[35] L. Zhao, X. Guo, C. Liu, Y. Sun, M. M. T. Loy, and S. Du, Optica 1, 84 (2014).

[36] Z. Han, P. Qian, L. Zhou, J. F. Chen, and W. Zhang, Sci. Rep. 5, 9126 (2015).

[37] S. E. Harris, Phys. Today 50, 36 (1997).

[38] C. Belthangady, S. Du, C.-S. Chuu, G. Y. Yin, and S. E. Harris, Phys. Rev. A 80, 031803(R) (2009).

[39] P. Kolchin, C. Belthangady, S. Du, G. Y. Yin, and S. E. Harris, Phys. Rev. Lett. 101, 103601 (2008).

[40] J. F. Chen, S. Zhang, H. Yan, M. M. T. Loy, G. K. L. Wong, and S. Du, Phys. Rev. Lett. 104, 183604 (2010).

[41] Y.-W. Cho, K.-K. Park, J.-C. Lee, and Y.-H. Kim, Phys. Rev. Lett. 113, 063602 (2014).

[42] L. Zhao, X. Guo, Y. Sun, Y. Su, M. M. T. Loy, and S. Du, Phys. Rev. Lett. 115, 193601 (2015).

[43] C. K. Hong, Z. Y. Ou, and L. Mandel, Phys. Rev. Lett. 59, 2044 (1987).

[44] S. Zhang, J. F. Chen, C. Liu, S. Zhou, M. M. T. Loy, G. K. L. Wong, and S. Du, Rev. Sci. Instrum. 83, 073102 (2012).

[45] See the Supplemental Material at http://link.aps.org/ supplemental/10.1103/PhysRevLett.117.013602 for a detailed derivation, which includes Ref. [46].

[46] M. O. Scully and M.S. Zubairy, Quantum Optics (Cambridge University Press, Cambridge, England, 1997).

[47] S. Du, J. Wen, and M. H. Rubin, J. Opt. Soc. Am. B 25, C98 (2008). 